\title{
Reversible and irreversible resistance changes for gamma-ray irradiation in silver-diffused germanium telluride
}

\author{
Hyoseong Park ${ }^{1} \cdot$ Tatsuya Watanabe $^{1} \cdot$ Isao $_{\text {Yoda }}{ }^{2} \cdot$ Yoshinori Shohmitsu $^{3} \cdot$ Shigeo Kawasaki $^{3} \cdot$ Toshihiro Nakaoka $^{1}$ (D)
}

Received: 15 July 2020 / Accepted: 19 November 2020 / Published online: 30 November 2020

(c) Springer Nature Switzerland AG 2020

\begin{abstract}
We report on the irreversible and reversible resistance changes for $\gamma$-ray irradiation in amorphous GeTe thin films with Ag electrodes. The $\gamma$-ray irradiation at a dose of $1 \mathrm{kGy}$ irreversibly decreased the DC resistance by two orders of magnitude. The irreversible resistance change was caused by the formation of a conductive region that consisted of $\mathrm{Ag}$-Te compounds. In-situ real-time DC resistance and AC impedance measurements revealed reversible variations in several electrode structures with $D C$ resistances ranging widely from about $10 \mathrm{k} \Omega$ to about $5 \mathrm{M} \Omega$. The $D C$ resistance decreased by 2-5\% with a time constant of about 3-7 min following the $\gamma$-ray irradiation with a dose rate of $0.5-2 \mathrm{kGy} / \mathrm{h}$, and recovered on interruption. The AC impedance measurement was analyzed with a simple equivalent circuit consisting of a parallel RC circuit of the Ag-diffused GeTe matrix, connected serially to the interface resistance. The interface resistance and the capacitance of the matrix exhibited a fast reversible variation, which is explained by trapping and detrapping of carriers in the charged defects formed by the Ag re-diffusion. The resistance of the matrix showed a slow reversible variation with a time constant of $7 \mathrm{~min}$, similar to the DC resistance. The slow reversible variation is attributed to the growth and dissolution of the conductive region caused by the Ag re-diffusion.
\end{abstract}

Keywords $\gamma$-ray Radiation sensor $\cdot$ Electrochemical reaction $\cdot$ GeTe $\cdot$ Silver chalcogenides

\section{Introduction}

Germanium chalcogenides have high radiation tolerance and have been used for radiation-hard nonvolatile memories [1-3], which are in high demand, especially by the space community. The resistances of both the crystalline and amorphous states of nonvolatile phase change memories using $\mathrm{Ge}_{2} \mathrm{Sb}_{2} \mathrm{Te}_{5}$ were unchanged by $\gamma$-ray up to $20 \mathrm{kGy}$ [3]. The radiation hardness arises from the structural recovery of the amorphous network after exposure, as well as the memory operation mechanism based on the phase change rather than the electric charge as in floating gate memories. The structural recovery and the specific structural rearrangements in the local atomic geometry were demonstrated by ab initio molecular-dynamics simulations on $\mathrm{Ge}_{2} \mathrm{Sb}_{2} \mathrm{Te}_{5}$ [4]. The radiation hardness and the structural recovery have also been studied intensively in Ag-doped Ge-chalcogenides. Suppression of the crystallization for $120 \mathrm{MeV} \mathrm{Ag}^{9+}$ heavy ion irradiation was reported in Ag-doped $\mathrm{Ge}_{2} \mathrm{Sb}_{2} \mathrm{Te}_{5}$ [5]. Healing of the damage to the silver chalcogenide network after $\gamma$-ray radiation, and remarkable reversibility upon annealing were demonstrated by an atomic simulation of Ag-doped $\mathrm{GeSe}_{3}$ [6].

Irradiation of $\mathrm{Y}$-ray onto Ag-doped Ge-chalcogenides induces the diffusion of silver ions. Resistance variation due to the diffusion has been applied to compact radiation dosimeters, which have attracted great interest due to their potential for reactor dosimetry, radiation chemistry, and clinical dosimetry. Successful sensing over

Toshihiro Nakaoka, nakaoka@sophia.ac.jp | ${ }^{1}$ Faculty of Science and Technology, Sophia University, Tokyo 102-8554, Japan. ${ }^{2}$ Tokyo Institute of Technology, Tokyo 152-8550, Japan. ${ }^{3}$ Japan Aerospace Exploration Agency, Sagamihara, Kanagawa 252-5210, Japan. 
a wide range of absorbed doses between $10^{3} \mathrm{~Gy}$ and $10^{7} \mathrm{~Gy}$ was demonstrated in Ag-doped Ge-S and Ge-Se systems [7-14]. However, the studies focused on the resistance variation between before and after $\mathrm{Y}$-ray irradiation. To the best of our knowledge, in-situ real-time measurements of the resistance variation, which can be applied to dose rate monitors, have not been reported yet in Ag-chalcogenide systems. In this work, we have performed in-situ real-time resistance measurement on amorphous germanium telluride (GeTe) thin films with Ag electrodes under $\gamma$-ray irradiation, and have observed both irreversible and reversible resistance variations. The causes of the irreversible resistance variations were revealed by time-of-flight secondary ion mass spectrometry (TOF-SIMS) measurements. The resistance was reduced irreversibly by growth of the conductive region that consisted of Ag-Te compounds, and it was increased by oxidation of $\mathrm{Ge}$ and $\mathrm{Te}$, which was distributed differently. Reversible resistance variation was decomposed into fast and slow components by analysis of the in-situ AC conduction measurement. A fast reversible variation was found in the interface resistance and the capacitance of the Ag-diffused GeTe matrix in the equivalent circuit that successfully fitted the complex impedance. The time constant of the fast variation was less than the frequency sweep time required to obtain the resistance and capacitance. A slow reversible variation was found in the resistance of the Ag-diffused GeTe matrix in the equivalent circuit extracted from the in-situ $A C$ measurement and the resistance of the in-situ DC measurement. The time constant of the slow variation was 3-7 min for the dose rate of $0.5-2 \mathrm{kGy}$.

The time constant is in the middle of the previously reported two types of "reversible" processes of Ag diffusion: we use the term "reversible" for the process that returns a state to the original when external fields such as $\gamma$-ray, UV-light, or electric fields are removed. One type is a very slow process where the recovery requires thermal annealing for several days at room temperature or annealing for about 10 mins at around half of the glass transition temperature. Two examples of the very slow process are the $\gamma$-ray induced photo-doping observed in the Ag-Ge-Se system $[9,12]$ and the photo-deposition in the Ag-Ge-S system [15, 16]. In these processes, the diffused or deposited Ag atoms can be reversed or dissolved back into the matrix by long-term or high temperature annealing. The other type is a very fast process of recovery to the original state within about $100 \mathrm{nsec}$. A typical example is the volatile switching found in filament-based selectors [17-19]. Our study of the middle rate of reversible variation may contribute to a unified understanding of these reversible changes.

\section{Materials and methods}

A $50 \mathrm{~nm}$ thick GeTe film was deposited on a thermally oxidized $\mathrm{Si}$ substrate $\left(300 \mathrm{~nm} \mathrm{SiO}_{2}\right.$ on (001) Si) by RF magnetron sputtering, using a GeTe target $(99.99 \%$ purity) with no intentional heating. The film was amorphous, and the composition was $\mathrm{Ge}_{50.8} \mathrm{Te}_{49.2}$, evaluated by inductively coupled plasma mass spectroscopy. The composition was reproduced under the condition that the plasma discharge power was $50 \mathrm{~W}$, the argon flow rate was $30 \mathrm{sccm}$, the pressure was $0.7 \mathrm{kPa}$, and the deposition rate was $2 \AA / \mathrm{s}$. On top of the film, a $50 \mathrm{~nm}$ thick Ag layer was deposited by electron-beam evaporation and was patterned to form electrodes by conventional photolithography and lift-off.

The DC resistance was obtained from the currentvoltage $(I-V)$ characteristics measured with an HP4142B semiconductor parameter analyzer. The AC complex impedance was measured with an LCR meter using a sinusoidal input over a range of frequencies from $1 \mathrm{kHz}$ to $100 \mathrm{kHz}$. The signal acquisition time for a frequency was about $2 \mathrm{~s}$, and the frequency sweep took about $2 \mathrm{~min}$. For the in-situ measurements under $\gamma$-ray irradiation, each Ag electrode was covered by a $10 \mathrm{~nm}$ thick Pt layer to prevent degradation of the electrodes due to $\mathrm{Ag}$ diffusion, and they are denoted as Ag/Pt. The Ag/Pt and Pt electrodes had Ti/Au bonding pads for wire bonding to a package onto which each device was mounted with conductive epoxy. The package was connected to the measurement system by long low-noise triaxial cables. The gamma-ray source was ${ }^{60} \mathrm{Co}$, and the radiation dose rate was controlled by the distance from the radiation source to the devices. The irradiation was performed at room temperature in an ambient atmosphere. We employed dose rates in the range of $0.1-2 \mathrm{kGy} / \mathrm{h}$. In the dose rate range, the results were qualitatively similar, and a common mechanism seems to be responsible, as we show later. Dose rate monitors operating in this dose-rate range are expected to have a wide range of applications. For example, the dose rates for radiation therapy are typically $0.1-0.4 \mathrm{kGy} / \mathrm{h}$ on a medical linear accelerators and a cobalt-60 machine [20].

\section{Results and discussion}

\subsection{Irreversible Ag diffusion and resistance variation}

The measurements of irreversible resistance variation were measured after the devices had been stored or 
annealed at room temperature in a vacuum desiccator in a dark place for 1 week after the $\gamma$-ray irradiation for $10 \mathrm{~h}$ at various dose rates with no bias applied to the electrodes. The 1 week storage or room temperature annealing was performed to focus on irreversible processes because in $\mathrm{Ag} / \mathrm{Ge}-\mathrm{Se}$ system Ag diffusion returning to its original state over $75 \mathrm{~h}$ after $\mathrm{\gamma}$-ray irradiation was reported. The resistances of the devices with a lateral electrode configuration were shown in the inset in Fig. 1 a. Six electrode pairs were measured for each irradiation dose. The irradiation at a dose rate of $0.1 \mathrm{kGy} / \mathrm{h}$ or a dose of $1 \mathrm{kGy}$ reduced the resistance by two orders of magnitude on average, as shown in Fig. 1a, although the amount of reduction was distributed reflecting Ag diffusion variation. On increasing the radiation dose further, the resistance increased slowly and became saturated around $10 \mathrm{kGy}$. To investigate the cause of the irreversible resistance variation, TOF-SIMS measurements were performed. After the $\gamma$-ray irradiation at the same doses as those used for the resistance measurements, $6 \mathrm{~nm}$ of the top surface layer was removed by $\mathrm{Bi}$ ion beam etching, and then the surface near an Ag electrode was analyzed by using $C s$ ion beam sputtering. The intensities of the secondary ions of $\mathrm{Ag}^{3-}, \mathrm{AgTe}^{-}, \mathrm{GeTe}^{2-}, \mathrm{Ge}_{2} \mathrm{O}_{5}{ }^{-}$, $\mathrm{TeO}_{3}{ }^{-}$, and $\mathrm{O}_{2}{ }^{-}$shown in Fig. $1 \mathrm{~b}$ represent the number of structural units having respectively $\mathrm{Ag}-\mathrm{Ag}$ bonds, $\mathrm{Ag}-\mathrm{Te}$ bonds, Ge-Te bonds, Ge-O bonds, Te-O bonds, and O-Obonds in the sputtered area.

The photomicrograph at $0 \mathrm{kGy}$, or before irradiation shows a bright contrast of the deposited Ag electrode region and a dark contrast region of about $50 \mu \mathrm{m}$ wide surrounding the electrode. The TOF-SIMS image of Ag-Ag bonds at $0 \mathrm{kGy}$ shows that the deposited $\mathrm{Ag}$ electrode region consisted of $\mathrm{Ag}-\mathrm{Ag}$ bonds before $\mathrm{\gamma}$-ray irradiation. In other words, the Ag atoms in the bright electrode region were unreacted. The TOF-SIMS images of $\mathrm{Ag}-\mathrm{Te}$ bonds and Ge-Te bonds show that the dark region has a larger number of structural units with $\mathrm{Ag}$-Te bonds, and a smaller number of structural units with Ge-Te bonds than the other region. The result demonstrates that the dark region was the Ag-diffused region that consisted of $\mathrm{Ag}-\mathrm{Te}$ compounds formed by the electrochemical reaction of Ag, consuming GeTe. The Ag-Te compounds are consistent with our previous works. Energy-dispersive $X$-ray spectroscopy on the same structure showed that the average composition ratio of the Ag-diffused region was roughly

(a)

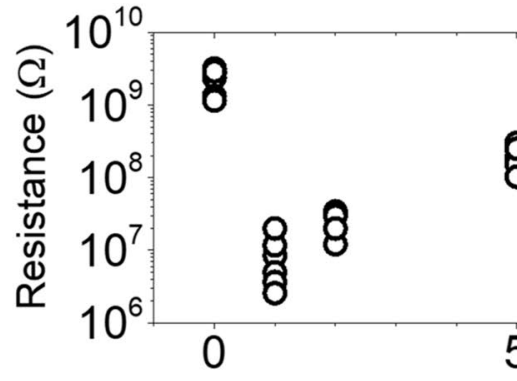

(b)

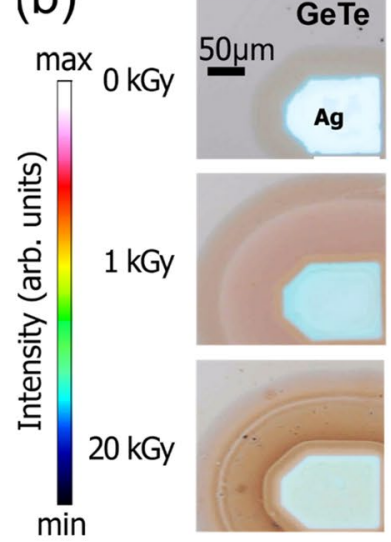

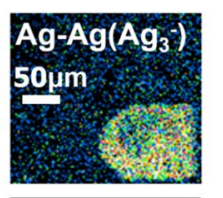
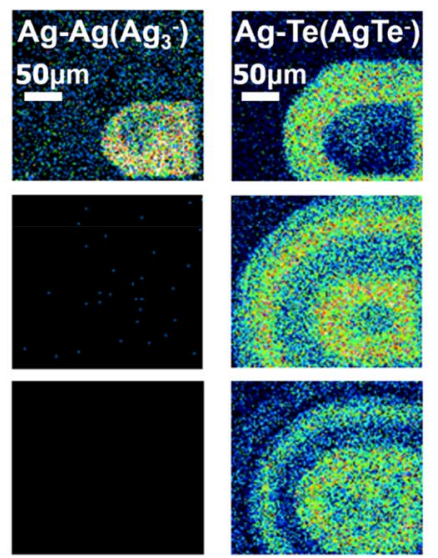

Fig. 1 (a) Resistances of the devices with an electrode configuration shown in the inset, measured at $0.1 \mathrm{~V}$, plotted as a function of the $\mathrm{\gamma}$-ray dose. (b) Photomicrographs and TOF-SIMS mapping images of secondary molecular ions of $\mathrm{Ag}^{3-}, \mathrm{AgTe}^{-}, \mathrm{GeTe}^{2-}, \mathrm{Ge}_{2} \mathrm{O}_{5}{ }^{-}$,

$\mathrm{TeO}_{3}{ }^{-}$, and $\mathrm{O}_{2}{ }^{-}$, which were sputtered from the device surfaces, at the doses of $0 \mathrm{kGy}, 1 \mathrm{kGy}$, and $20 \mathrm{kGy}$. The intensities are normalized to the maximum counts 
Ag:Ge:Te = 1:1:1 [21]. Transmission electron microscope and electron diffraction measurements showed that the electrochemical reaction between $\mathrm{Ag}$ and GeTe formed Ge-rich and Ag-Te-rich amorphous regions, phase-separated with a size of about $10-30 \mathrm{~nm}$, as well as nanocrystals of the monoclinic phase of $\mathrm{Ag}_{2} \mathrm{Te}$ [22]. Here we note that the $\mathrm{Ag}$ atoms can diffuse not only in the amorphous regions but also in the region of the monoclinic crystalline $\mathrm{Ag}_{2} \mathrm{Te}$; the diffusion constant of $\mathrm{Ag}$ in the monoclinic $\mathrm{Ag}_{2} \mathrm{Te}$ was reported to be around $10^{-4} \mathrm{~cm}^{2} \mathrm{~s}^{-1}$ at $110^{\circ} \mathrm{C}$ [23].

The TOF-SIMS image at $1 \mathrm{kGy}$ shows that the unreacted Ag region of Ag-Ag bonds almost disappeared. Instead, the Ag-diffused region of a dark contrast consisted of a large number of Ag-Te bonds and a reduced number of Ge-Te bonds expanded to about $100 \mu \mathrm{m}$ in width. The expanded Ag-diffused region determines the resistance at $1 \mathrm{kGy}$ of devices with the electrode separation of $60 \mu \mathrm{m}$ because the width was larger than the separation. The reduced resistance at $1 \mathrm{kGy}$ is attributed to the lower resistivity of the expanded Ag-diffused region than that of unreacted GeTe. There are three possible causes of the lower resistivity: the material resistivity of Ag-Te compounds, the inclusion of the nanocrystals of monoclinic $\mathrm{Ag}_{2} \mathrm{Te}$, which is a low bandgap semiconductor with bulk resistivity less than $10^{-2} \Omega \cdot \mathrm{cm}$ [24], and Ag diffusion-induced formation of the impurity bands that induces insulator-metal transition as reported in a $\mathrm{GeSe}_{3}$ :Ag glass [25]. The expansion of Ag diffusion accompanies the emergence of the Ge-O region, which has a large number of $\mathrm{Ge}-\mathrm{O}$ bonds in the form of a curved swath surrounding the electrode. The $\mathrm{Ge}-\mathrm{O}$ region is attributed to $\mathrm{O}$-rich $\mathrm{Ge}$ oxides because the region had more $\mathrm{O}-\mathrm{O}$ bonds than the unreacted regions. The emergence of the Ge-O region within the Ag-diffused region suggest that the region was formed by oxidation of the $\mathrm{Ge}$ atoms precipitated during the Ag-Te formation. The expansion of Ag diffusion also accompanies the emergence of the Te-O region, which has a large number of $\mathrm{Te}-\mathrm{O}$ bonds in the electrode area. The Te-O region is attributed to O-rich Te oxides because the region had more O-O bonds. The fact that there were fewer Ge-Te bonds in the region than the other regions suggests that the Te oxides were formed by oxidation of the Te atoms left behind after diffusion of plenty of Ag atoms of the electrode.

The TOF-SIMS image at $20 \mathrm{kGy}$ shows that the Ag-diffused region observed as the dark region of the photograph and as the region of Ag-Te compounds was very similar in size to that at $1 \mathrm{kGy}$. This means that the expansion of the Ag-diffused region was saturated around $1 \mathrm{kGy}$. The number of Ag-Te bonds decreased in the entire device and that of Ge-Te bonds also decreased especially outside the Ag-diffused region. Instead, the number of $\mathrm{Ge}-\mathrm{O}$ and $\mathrm{O}-\mathrm{O}$ bonds increased. The oxidation outside the
Ag-diffused region indicates that the $\gamma$-ray irradiation at 20 kGy directly broke the Ge-Te bonds, and the environmental oxygen formed Ge-O bonds preferentially rather than Te-O bonds.

On the basis of the irreversible Ag diffusion and the resistance variation we propose the following processes. The $\gamma$-ray irradiation up to $1 \mathrm{kGy}$ promotes the $\mathrm{Ag}$ diffusion and expands the Ag-diffused region to a width of nearly $100 \mu \mathrm{m}$. An Ag atom preferentially enters the Ge site [26] of the Ge-Te bond, and forms an Ag-Te bond. The leftover $\mathrm{Ge}$ atoms precipitate to form an amorphous region with weaker bonds than the original Ge-Te bonds. As the Ag diffusion proceeds, the $\mathrm{Ag}$ atoms separate from the $\mathrm{Ag}$-Te bonds, and leave Te atoms behind with weakened bonds by forced bond-switching. In particular, plenty of Ag atoms of the electrode diffuse through the Te atoms just below the electrode, leaving many Te atoms with weakened bonds in their place. $\gamma$-ray irradiation breaks the weakened bonds of the precipitated $\mathrm{Ge}$ and those of the Te atoms left after mediating Ag diffusion, and form dangling bonds on the surface to be connected with oxygen in the environment. As a result, Ge oxide is formed in the Ag-Te region while Te oxide is formed in the region where the Ag electrodes had been present. The amount of $\mathrm{Ag}$ atoms in the electrode of a thickness of $50 \mathrm{~nm}$ limits the further expansion of the diffused region even when the dose is increased above $1 \mathrm{kGy}$. Instead, the $\gamma$-ray of the dose above $1 \mathrm{kGy}$ directly breaks the Ge-Te and Ag-Te bonds to form $\mathrm{Ge}$ and Te oxides in the overall surface area of the device. The oxidation increases the resistance until the oxidation saturates. We note that the significant effect of the oxidation on the resistance obscures the effect of partial crystallization that may occur above 6 kGy [27].

\subsection{Reversible impedance variation}

Reversible resistance variation was studied with a GeTe film having a lateral $\mathrm{Ag} / \mathrm{Pt}-\mathrm{Pt}$ electrode pair with a wide separation of $100 \mu \mathrm{m}$, as shown in the inset of Fig. 2a. Figure 2a shows the $I-V$ characteristics without $\gamma$-ray irradiation. The sweep rate-dependent peaks around $\pm 0.5-1.0 \mathrm{~V}$ arise from the redox reaction, indicating that the system allows ionic conduction of $\mathrm{Ag}$ and involves a repetitive faradaic electrode reaction [28]. We employed the in-situ AC impedance measurement on the device using a sinusoidal input voltage restricted to $0.3 \mathrm{~V}$ to focus on the resistance of the electronic contribution rather than that of the ionic contribution. We note here that unlike the resistance measurements in Fig. 1, the in-situ AC impedance measurement and the following in-situ resistance measurements were performed after $I-V$ measurements to check the devices before $\gamma$-ray irradiation. The $I-V$ measurement reduced the base resistance, especially in the first voltage application 

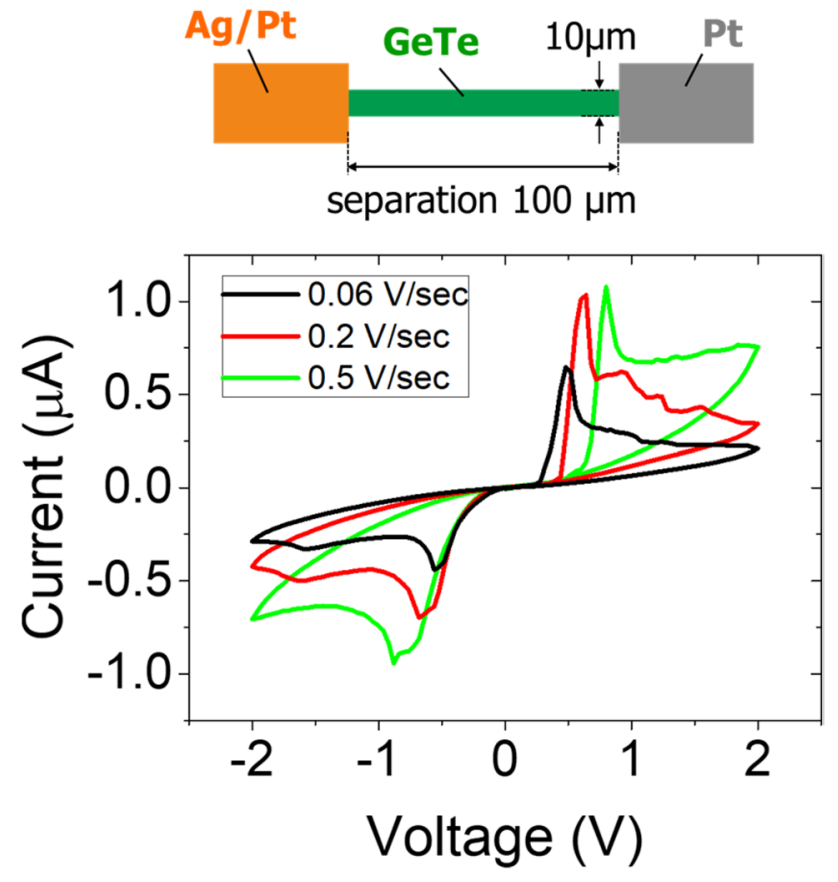

Fig. 2 Schematic of the device structure having an Ag/Pt-Pt lateral electrode pair with a separation of $100 \mu \mathrm{m}$ used for reversible resistance measurement and the $I-V$ characteristics measured at three sweep rates

because of the redox processes of forming Ag ions [29] that led to precursory filament growth, which occurred even less than the SET voltage [21]. Consequently, the base resistances were lower than those expected from Fig. 1, and therefore the initial rapid drop of the resistance due to the $\gamma$-ray irradiation less than $1 \mathrm{kGy}$ was insignificant.

Figure 3a shows the Nyquist plots of the impedance with and without the $\gamma$-ray irradiation of a device having the electrode structure shown in Fig. 2. The $y$-ray was irradiated at a dose rate of $0.5 \mathrm{kGy} / \mathrm{h}$ for $20 \mathrm{~min}$ and interrupted for $20 \mathrm{~min}$. Each Nyquist plot shows a capacitive behavior with an arc of semicircle similar to the Ag-doped $\mathrm{Ge}_{17} \mathrm{Te}_{83}$ [29]. The capacitive behavior is well fitted by a simple equivalent circuit consisting of a resistor $R_{s}$ connected in series with a parallel resistor-capacitor $\left(R_{p}-C_{p}\right)$, where $R_{s}$ represents the interface resistance, while $R_{p}$ and $C_{p}$ are the resistance and the capacitance of the AgGeTe matrix. A slight deviation from each fitting curve in the low-frequency region is attributed to the contribution from the electrode polarization $[30,31]$ or the slow ionic dynamics that modulate the electronic current [32, 33]. The obtained $R_{p}, R_{s}$ and $C_{p}$ are plotted in Fig. $3 b$ as a function of time. Both the first and the second irradiations decreased $R_{p}$ by $\left(R_{\text {without }}-R_{\text {under }}\right) / R_{\text {without }}=4.4 \%$, and the interruptions returned $R_{s}$ to almost the initial value. The response time was about $7 \mathrm{~min}$ for both the irradiation and interruption. In contrast to the slow variation of $R_{p}$, the variation of $R_{s}$ and $C_{p}$ was fast, had the opposite direction of change, and showed a difference between the 1 st and the 2 nd irradiation. $R_{s}$ and $C_{p}$ varied very similarly. The first $\gamma$-ray irradiation raised the values, and then immediately returned them to almost their pre-irradiation (a)

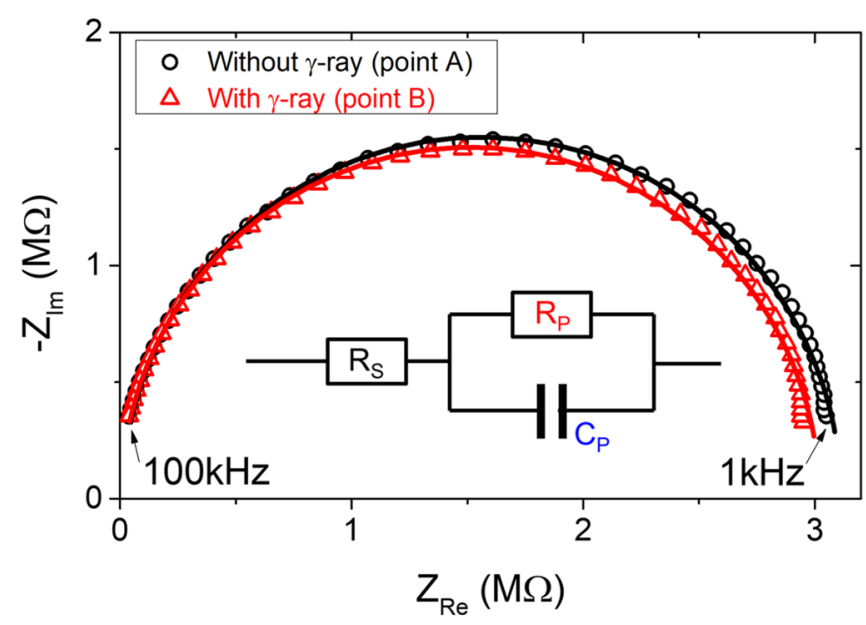

Fig. 3 (a) Impedance spectra of the device having the electrode structure shown in Fig. 2 , with and without $\gamma$-ray irradiation. The spectra are fitted with the equivalent circuits shown in the inset, where $R s$ is the total interface resistors, $R_{p}$ and $C_{p}$ are the resistance (b)

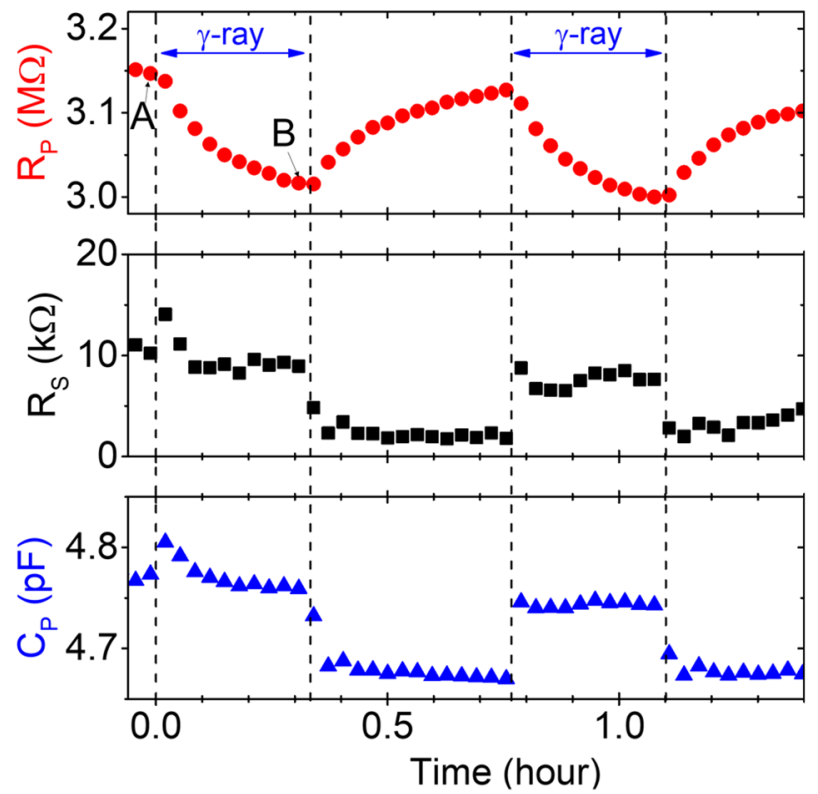

and capacitor of the Ag-diffused GeTe matrix. (b) $R_{p}, R_{s^{\prime}}$ and $C_{p}$ plotted as a function of time under the $\gamma$-ray irradiation at $0.5 \mathrm{kGy} / \mathrm{h}$ for $20 \mathrm{~min}$ and without the $\mathrm{y}$-ray for $20 \mathrm{~min}$. The cycle was repeated twice 
values in a few minutes, after which the value remained almost constant during the irradiation. The interruption of the irradiation dropped the values to less than the preirradiation values with a response time less than the sweep time of $2 \mathrm{~min}$. The second irradiation raised the values to their pre-irradiation levels, and the second interruption dropped them to the values after the first irradiation. Thus, the first irradiation reduced the base values of the $R_{s}$ and $C_{p}$ irreversibly, and the second irradiation induced reversible switching of the values.

\subsection{Reversible DC resistance variation}

Following the impedance measurement, we performed the in-situ DC resistance measurement on the same device. The $\mathrm{\gamma}$-ray irradiation at $0.5 \mathrm{kGy} / \mathrm{h}$ for $20 \mathrm{~min}$ reduced the resistance by about $2 \%$ and the interruption for $20 \mathrm{~min}$ recovered and increased it, as shown in Fig. 4. The time constant was about $3 \mathrm{~min}$, both for the reduction and the increase. During the irradiation, the base resistance gradually irreversibly increased, possibly due to oxidation.

Next, we investigated reversible variations in the devices of relatively low resistances. Two electrode configurations having a narrow separation of $10 \mu \mathrm{m}$ were used: one was a simple electrode pair and the other was an interdigital electrode pair as shown in Fig. $5 a$ and $b$. The voltage sweep was limited to $\pm 0.1 \mathrm{~V}$ because the $\mathrm{Ag}$ filaments can be easily connected in the narrow electrode devices. Because in this voltage range the $I-V$ curves were almost linear, the resistances at $0 \mathrm{~V}$ are plotted. The two narrow electrode configurations resulted in higher resistances than those expected assuming a uniform current

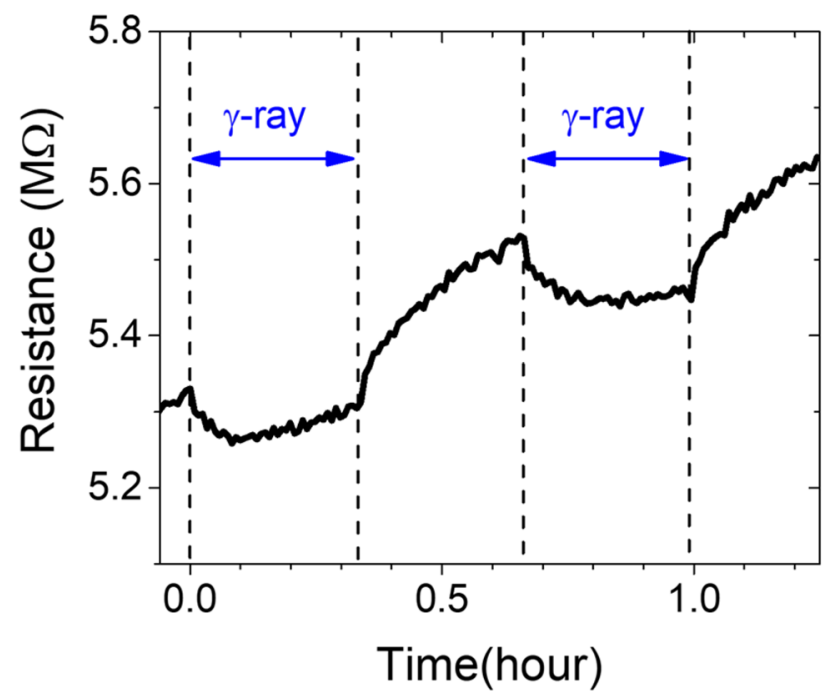

Fig. 4 DC resistance variations under and without $\gamma$-ray irradiation at a dose rate of $500 \mathrm{~Gy} / \mathrm{h}$, measured at $0.1 \mathrm{~V}$ after the impedance measurement density and the same resistivity as that of the $100 \mu \mathrm{m}$ wide electrode pair in Fig. 4. The resistance of the simple electrode pair was about $80 \mathrm{k} \Omega$, which was about three times larger than the value of $5.5 / 190 \mathrm{M} \Omega$ expected from the value of $5.5 \mathrm{M} \Omega$ of the wide electrode device, 1/10th the separation and 1/19th the width. The resistance of the interdigital electrode was about $10 \mathrm{k} \Omega$, which was about four times larger than the value of $5.5 / 2100 \mathrm{M} \Omega$ expected from the value of $5.5 \mathrm{M} \Omega, 1 / 10$ th the separation and $1 / 210$ th the total width. The higher resistance values are consistent with the composition distribution after $\mathrm{Ag}$ diffusion with fewer Ag-Te bonds and more $\mathrm{Ge}-\mathrm{O}$ and Te-O bonds near the electrode than in the outer area, as shown in Fig. 1, resulting in a higher resistivity near the electrode than the other area. The result suggests that in the measured devices current flowed not through a specific path such as filaments or an interface, but rather flowed over the entire device area.

In the in-situ resistance measurements in the narrowelectrode devices, current fluctuations often occurred at the beginning of irradiation. To suppress this, the measurements were performed after three cycles of 3-min irradiation. The $\gamma$-ray irradiation on the device with the simple electrode pair at a dose rate of $2 \mathrm{kGy} / \mathrm{h}$ for $1 \mathrm{~h}$ decreased the resistance by about $5 \%$, and the interruption for $1 \mathrm{~h}$ recovered and increased it, as shown in Fig. 5a. The $y$-ray irradiation on the device with the interdigital electrode pair at a dose rate of $2 \mathrm{kGy} / \mathrm{h}$ for $1 \mathrm{~h}$ decreased the resistance by about $4 \%$, and the interruption for $1 \mathrm{~h}$ recovered and increased it, as shown in Fig. 5b. In both devices, the reversible resistance variations were repeated three times with a time constant of about $7 \mathrm{~min}$. Thus, although the surface area of the interdigital electrode pair was more than ten times larger than the simple pair, the $\gamma$-ray induced resistance variations were very similar. The independence of the electrode configuration shows that the reversible resistance variation occurred not at a specific location or interface, but over the entire device area.

\subsection{Mechanism of slow reversible resistance variation}

The slow reversible variation observed in the $\mathrm{DC}$ resistance and $R_{p}$ had time constants of 3-7 min for the $\gamma$-ray irradiation and interruption. The time constants suggest that the reversible variation involves Ag diffusion rather than the carrier dynamics. Three representative phenomena of reversible Ag diffusion have been reported, induced by $\gamma$-ray, UV-light, and an external electric field. The first one is the $y$-ray-induced Ag diffusion that can be reversed by long-term annealing. A recovery of the resistance after room temperature annealing for $75 \mathrm{~h}$ was reported in $\mathrm{Ag} / \mathrm{Ge}_{30} \mathrm{Se}_{70}$. $[9,12]$. The recovery was 
Fig. 5 Schematic and resistance variations under and without $\gamma$-ray irradiation of the devices having (a) a simple lateral electrode pair with a separation of $10 \mu \mathrm{m}$ and a width of $190 \mu \mathrm{m}$ and (b) an interdigital electrode pair with a separation of $10 \mu \mathrm{m}$ and a width of $390 \mu \mathrm{m}$
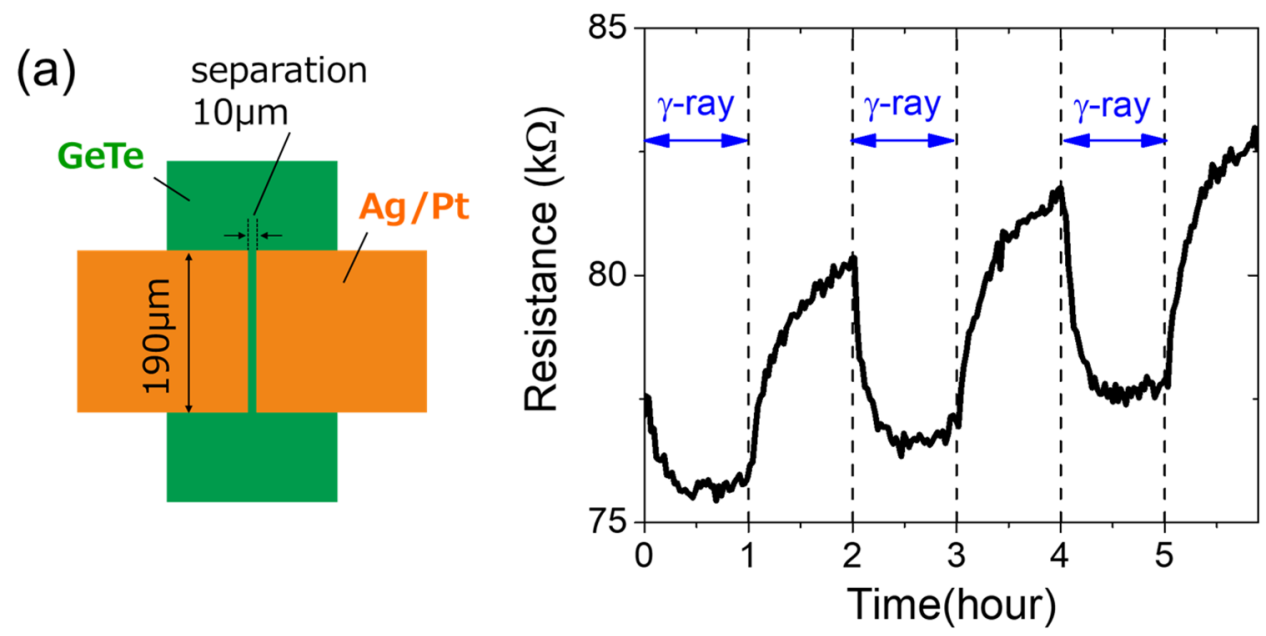

(b)

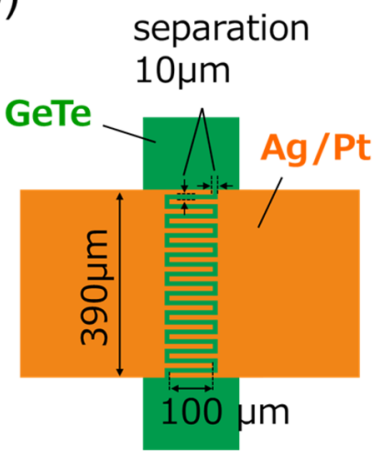

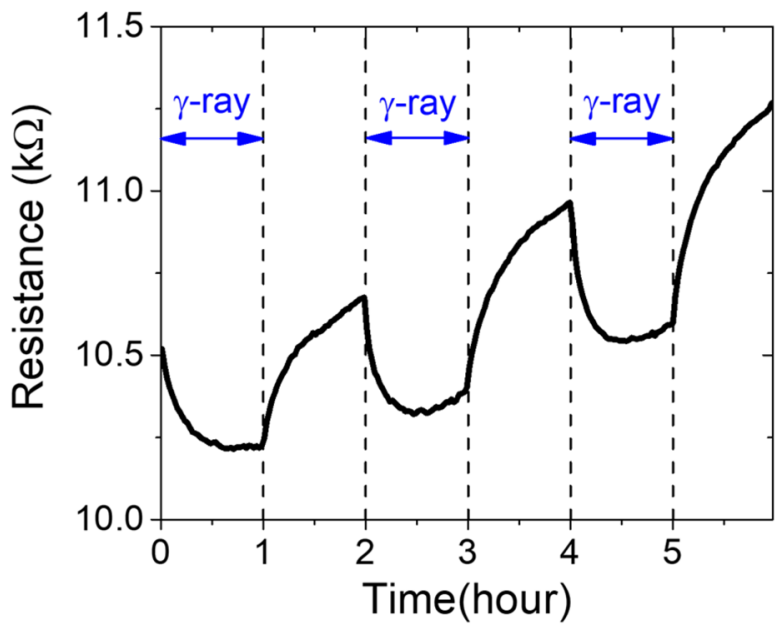

explained as being due to the built-in electric field that was reversed during $\gamma$-ray irradiation by different distribution between excited electrons and holes. The second one is photo-deposition, reported in the Ag-Ge-S system, in which the Ag conductive region was formed by a UV light and was dissolved or reversed into the matrix by thermal annealing, for example, at $100{ }^{\circ} \mathrm{C}$ for 10 min $[15,16]$. The photo-deposition was explained by assuming that the amorphous Ag-Ge-S was oversaturated with $\mathrm{Ag}$ and metastable at room temperature. After illumination, the metastable system approached an equilibrium to segregate excess $\mathrm{Ag}$ and was dissolved by annealing due to increased solubility. The third one is the volatile resistance switching reported in $\mathrm{Ag}-\mathrm{Ge}_{2} \mathrm{~S}$ $\mathrm{b}_{2} \mathrm{Te}_{5}$ and $\mathrm{Ag}-\mathrm{GeTe}_{8}$ [17-19], which is applied to selectors in a crosspoint memory array. An external voltage bias application induced electrodeposition of Ag-rich conductive filaments, and no voltage application dissolved the filaments to return to the original state. The reversible conductive filament growth was explained by the competition between the electrodeposition and the dissolution with the aid of chemical potential gradient and electrolyte nonstoichiometry; the external voltage determined which process predominated.

In light of these characteristics of Ag diffusion in the Ag chalcogenides, we propose the following mechanism for the $\gamma$-ray induced reversible variation of the DC resistance and $R_{p}$. The observation of the redox peaks in Fig. 2 arising from the electrochemical reaction of movable $\mathrm{Ag}$ ions demonstrated that the formation and dissolution of the conductive region are competitive even without $\gamma$-ray irradiation; in other words, the "irreversible" states, the resistances of which are shown in Fig. 1, result from the equilibrium of the competitive growth and dissolution except for the effect of oxidation. Irradiation by $Y$-ray produces electrons, holes, and $\mathrm{Ag}+$ ions. The positive and negative charges distribute differently, as in the process of photodoping [34]. We note that the distribution of excited electrons is rather important because GeTe is generally unintentionally $\mathrm{p}$-doped $\left(\sim 10^{21} \mathrm{~cm}^{-3}[35]\right)$, and the number of excited holes is much smaller than that of the intrinsic holes. Because the electron affinity of GeTe is $4.8 \mathrm{eV}$ [36] and the work function of polycrystalline $\mathrm{Ag}$ is $4.26 \mathrm{eV}$ [37], Fermi level aligning at equilibrium makes a downward 
band bending toward the Ag-GeTe interface. Therefore, negative charges are expected to move to the nearest electrode and positive charges are expected to move in the opposite direction. The dynamics of the excited carriers and ions makes the electric field distribution inside the device different from that without irradiation. The modified electric field distribution promotes re-diffusion of $\mathrm{Ag}^{+}$ions. The re-diffusion induces growth of the $\mathrm{Ag}$-Te conductive region and formation of the impurity bands that can lead to an insulator-metal transition to decrease the resistance. On the other hand, interruption of the $\gamma$-ray returns the balance between growth and dissolution to that before irradiation with the aid of chemical potential gradient and electrolyte nonstoichiometry. One cause of the chemical potential gradient is negative enthalpy of mixing between $\mathrm{Ag}$ and Te throughout the composition range of Ag-Te alloy, the minimum being $-20 \mathrm{~J} / \mathrm{mol}$ at the composition of $\mathrm{Ag}_{2} \mathrm{Te}[38,39]$. The recovery time of 3-7 min at room temperature, which is much shorter than $75 \mathrm{~h}$ in an $\mathrm{Ag} / \mathrm{Ge}_{30} \mathrm{Se}_{70}$ device, can be attributed to the characteristics of $\mathrm{Ag}$ in Te-based chalcogenides and the smallness of the structural variations that cause the resistance variation. In $\mathrm{Ag}-\mathrm{GeTe}_{8}$, and $\mathrm{Ag}-\mathrm{Ge}_{2} \mathrm{Sb}_{2} \mathrm{Te}_{5}, \mathrm{Ag}$ diffusion and dissolution were facilitated by non-bonded Te anions $[17,18]$ and longer Ag-Te bonds than other bonds [19]. In addition, the insulator-metal transition from amorphous to amorphous, as predicted by first-principles calculations of $\left(\mathrm{GeSe}_{3}\right)_{1-x} \mathrm{Ag}_{x}$ [25], may contribute to the resistance variation. The calculations showed that stable amorphous phases with $\sim 10^{8}$ times higher electronic conductivity existed with only a small structural difference. Thus, even very small structural changes can cause resistance changes of more than a few percent as observed.

\subsection{Mechanism of fast variation}

The Ag re-diffusion under $\gamma$-ray irradiation that induces the slow resistance variation of the $D C$ resistance and $R_{p}$ is the key to understanding the fast variation found in the interface resistance $R_{s}$ and the capacitance of the matrix $C_{p}$. We explain the fast variation by the trapping and detrapping of carriers in the charged defects formed by the Ag rediffusion. Generally, charged defects in amorphous chalcogenides forms localized states in the band tail regions, and play a significant role in the $D C$ and $A C$ conduction [40-42]. We assume that in the first $\gamma$-ray irradiation, the Ag re-diffusion forms charged defects in addition to the intrinsic ones, because defect formation due to metal atom diffusion in semiconductors has been commonly observed [43]. In chalcogenide glasses, many models have been presented for the conduction mechanism. Poole-Frenkel ionization, field-induced delocalization of tail states, spacecharge limited currents, optimum channel hopping in thin films, optimum channel field emission, percolation band conduction, and transport through crystalline inclusions are all candidate explanations for the conduction [44]. All models involve localized states or local potential minima. Even in space-charge limited model, localized states are responsible for the low mobility for space charge-limited current. Therefore, we assume charged defects near an electrode are responsible for the conduction of Rs, and the electronic states of neutral defects do not contribute to the conduction significantly. The increase of $R_{s}$ under $\gamma$-ray irradiation is explained by trapping of carriers in the charged defects, which neutralizes them, as shown in Fig. 6a. In particular, trapping of electrons would mainly be responsible for variation of Rs, because the number of excited holes is much smaller than that of the intrinsic holes in the unintentionally $\mathrm{p}$-doped GeTe. The recovery of $R_{s}$ is explained by detrapping process, which recharges them. The variation in $R_{s}$ for $\gamma$-ray irradiation and interruption is determined by that of the number of charged states as shown in Fig. 6b.

At the beginning of the first $\gamma$-ray irradiation, $R_{s}$ increases due to the trapping of carriers neutralizing the intrinsic charged defects. Then, $R_{s}$ is decreased by a gradual formation of the additional charged defects caused by the $\mathrm{Ag}$ diffusion. The increase in $\mathrm{R}_{\mathrm{s}}$ by the neutralization of charged defects and the decrease by the formation of additional charged defects almost cancel each other out and eventually saturate at a value close to the pre-irradiation one. When the irradiation is interrupted, detrapping of the carriers recharges the defects. The recharging decreases $R_{s}$ to less than the pre-irradiation value because the total number of charged defects after the first radiation is larger than that before irradiation due to the diffusion-induced charged defects. We assume that the re-diffusion of $\mathrm{Ag}$ in the second and subsequent $\gamma$-ray irradiation at the same dose rate follows the same path to generate no additional defects. Then, the $\gamma$-ray induced resistance variation becomes determined simply by the trapping and detrapping of the carriers in the charged defects, the number of which was fixed after the first irradiation. The trapping of the carriers generated by the second and the subsequent $\gamma$-ray irradiation neutralizes the charged defects to increase $R_{s}$ to the saturated value during the first irradiation, and the detrapping by the interruption recharges them to decrease $R_{s}$ to a value less than the pre-irradiation value.

The fast reversible variation of $C_{p}$ is explained by the trapping of carriers in the charged defects with a dielectric model of point charge defects in paraelectric perovskites, where the electric field from a charged point defect locally polarizes the surroundings to reduce its permittivity [45]. We apply this model to the charged defects of the Ag-GeTe for the following two reasons. First, a crystalline GeTe is a 
Fig. 6 (a) Schematic energy band diagram during and after $\gamma$-ray irradiation. Trap of electrons and holes neutralizes charged defects $\mathrm{D}^{+}, \mathrm{D}^{-}$into $D^{0}$. Detrap recharges them. (b) Schematic representation of the variation in number of charged states as a function of time for $\gamma$-ray irradiation and interruption

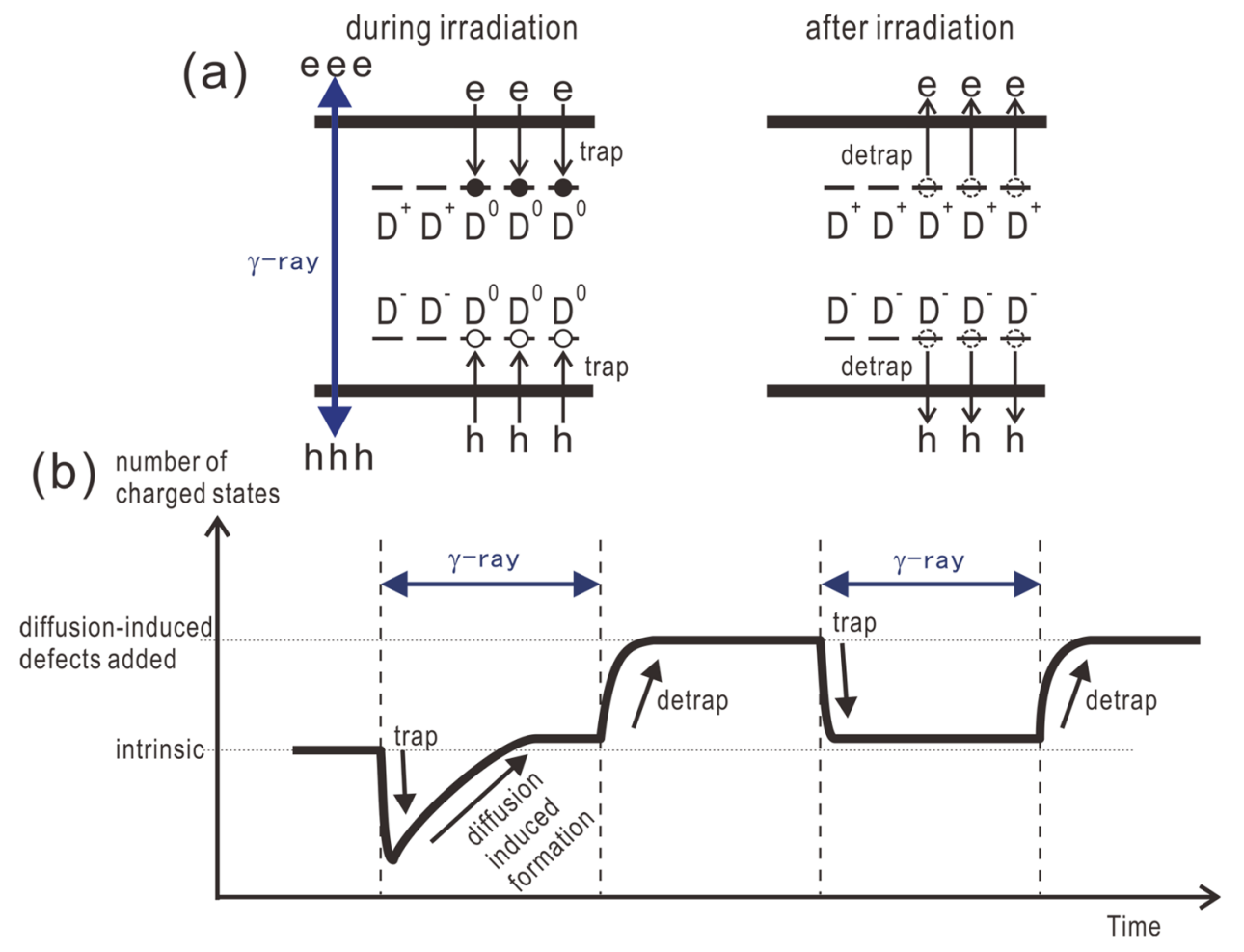

ferroelectric material with a ferroelectric-paraelectric transition like perovskites $[46,47]$. The ferroelectric properties of GeTe arise from the distortion of the unit cell [48], and persist even in colloidal nanocrystals of $5 \mathrm{~nm}$ in size [49]. Because the short-range order is generally maintained in the amorphous form, the model is applied to describe the surroundings of the charged defects in the amorphous matrix. Second, the model predicts a reduction of the capacitance when an external electric field is applied, by a process in which the electric field detraps the electrons trapped in the point defects and recharges the neutralized point defects. Such a reduced capacitance by applying an external electric field was reported in an amorphous Ag-doped $\mathrm{Ge}_{17} \mathrm{Te}_{83}$, which exhibited a similar capacitive impedance to our Ag-GeTe [28].

Because of the existence of intrinsic charged defects, the pre-irradiation value of $C_{p}$ is lower than the ideal defect-free value. Under the first $\gamma$-ray irradiation, the intrinsic charged defects capture the excited carriers and are neutralized to recover or increase $C_{p}$. $C_{p}$ is then decreased by the formation of additional charged defects by the re-diffusion of $\mathrm{Ag}$. The increase in $\mathrm{C}_{\mathrm{p}}$ by the neutralization of charged defects and the decrease by additional charged defects almost cancel each other out, and eventually saturate at a value close to the pre-irradiation one. When the irradiation is interrupted, the carriers are detrapped to recharge the defects. The recharging of the defects decreases $C_{p}$ to less than the pre-irradiation value because the total number of the charged defects after the first irradiation is larger than that before radiation by the diffusion-induced charged defects. In the second and subsequent $\gamma$-ray irradiation, the re-diffusion of Ag generates no additional defects, and the capacitance starts to exhibit reversible variation determined by the trapping and detrapping of the carriers in the charged defects. The trapping of carriers neutralizes the charged defects to increase $C_{p}$ to the value saturated during the first irradiation, and the detrapping during the irradiation interruption recovers the charged defects to decrease $C_{p}$ to a value less than the pre-irradiation value.

\section{Conclusions}

We have revealed both the irreversible and reversible $\mathrm{Ag}$ diffusion and the resistance for $\gamma$-ray irradiation in amorphous GeTe thin films with Ag electrodes. The TOF-SIMS and DC resistance measurements showed that the irreversible Ag diffusion formed a conductive region that consisted of Ag-Te compounds. The $\gamma$-ray irradiation up to $1 \mathrm{kGy}$ broke the weakened bonds of the $\mathrm{Ge}$ and Te atoms distributed differently. The $\mathrm{\gamma}$-ray of the dose above $1 \mathrm{kGy}$ directly broke the Ge-Te and Ag-Te bonds to form Ge and Te oxides in the entire device. The in-situ real-time DC resistance and $A C$ impedance measurements revealed reversible Ag re-diffusion, triggered by electric field modification due to the excited carriers and ions. The Ag rediffusion modified the conductive region, which induced 
the slow, reversible DC resistance variation with a time constant of 3-7 min. The Ag re-diffusion formed additional charged defects. Trapping and detrapping of excited carriers in the charged defects induced the fast reversible variations in $R_{s}$ and $C_{p}$ with a time constant less than the sweep time. We believe the observed reversible variation of the resistance and the impedance in a simple device structure paves the way toward the creation of a compact dose rate monitor with high radiation tolerance.

Acknowledgements This work was supported in part by MEXT KAKENHI Grant Numbers 18H01480, and by specially promoted academic research from Sophia University. Our research was partially preformed in the nano-electronics clean room in Institute of Space and Astronautical Institute (ISAS).

\section{Complaince with ethical standards}

Conflict of interest The authors declare that they have no conflict of interest.

\section{References}

1. Bernacki S, Hunt K, Tyson S, Hudgens S, Pashmakov B, Czubatyj W (2000) Total dose radiation response and high temperature imprint characteristics of chalcogenide based RAM resistor elements. IEEE Trans Nucl Sci 47:2528-2533. https://doi. org/10.1109/23.903803

2. Maimon J, Hunt K, Burcin L, Rodgers J (2003) Chalcogenide memory arrays: characterization and radiation effects. IEEE Trans Nucl Sci 50:1878-1884. https://doi.org/10.1109/tns.2003.82137 7

3. Liang-Cai W, Bo L, Zhi-Tang S, Gao-Ming F, Song-Lin F, Bomy C (2006) Total dose radiation tolerance of phase change memory cell with GeSbTe alloy. Chin Phys Lett 23:2557-2559. https://doi. org/10.1088/0256-307x/23/9/057

4. Konstantinou K, Lee TH, Mocanu FC, Elliott SR (2018) Origin of radiation tolerance in amorphous $\mathrm{Ge}_{2} \mathrm{Sb}_{2} \mathrm{Te}_{5}$ phase-change random-access memory material. Proc Natl Acad Sci 115:53535358. https://doi.org/10.1073/pnas.1800638115

5. Kanda N, Thakur A, Singh F, Singh A (2020) Effect of ion irradiation on the optical properties of Ag-doped $\mathrm{Ge}_{2} \mathrm{Sb}_{2} \mathrm{Te}_{5}$ (GST) thin films. Nucl Instrum Methods Phys Res, Sect B 467:40-43. https ://doi.org/10.1016/j.nimb.2020.01.025

6. Prasai K, Drabold DA (2014) Simulations of silver-doped germanium-selenide glasses and their response to radiation. Nanoscale Res Lett. https://doi.org/10.1186/1556-276x-9-594

7. Chen P, Ailavajhala M, Mitkova M, Tenne D, Sanchez Esqueda I, Barnaby H (2011) Structural study of Ag-Ge-S solid electrolyte glass system for resistive radiation sensing. 2011 IEEE Workshop on Microelectronics and Electron Devices. doi: https:// doi.org/10.1109/wmed.2011.5767271

8. Ailavajhala M, Mitkova M, Butt DP (2012) Simulation and process flow of radiation sensors based on chalcogenide glasses for in situ measurement capability. Phys Status Solidi C 9:24152419. https://doi.org/10.1002/pssc.201200197

9. Gonzalez-Velo Y, Barnaby HJ, Chandran A, Oleksy DR, Dandamudi P, Kozicki MN, Holbert KE, Mitkova M, Ailavajhala M, Chen $P$ (2012) Effects of Cobalt-60 gamma-rays on Ge-Se Chalcogenide glasses and Ag/Ge-Se test structures. IEEE Trans Nucl Sci 59:3093-3100. https://doi.org/10.1109/tns.2012.2224137
10. Mitkova M, Chen P, Ailavajhala M, Buttbe DP, Tennec DA, Barnabyd H, Esquedad IS (2013) Gamma ray induced structural effects in bare and $\mathrm{Ag}$ doped $\mathrm{Ge}-\mathrm{S}$ thin films for sensor application. J Non-Cryst Solids 377:195-199. https://doi.org/10.1016/j.jnonc rysol.2012.12.031

11. Dandamudi P, Kozicki MN, Barnaby HJ, Gonzalez-Velo Y, Mitkova M, Holbert KE, Ailavajhala M, Yu W (2013) Sensors based on radiation-induced diffusion of silver in Germanium Selenide glasses. IEEE Trans Nucl Sci 60:4257-4264. https://doi.org/10.1109/ tns.2013.2285343

12. Mitkova M, Butt D, Kozicki M, Barnaby H (2013) Chalcogenide glass radiation sensor. Mater Dev Design Device Test. https:// doi.org/10.2172/1082961

13. Ailavajhala M, Gonzalez-Velo Y, Poweleit C, Barnaby HJ, Kozicki MN, Butt DP, Mitkova M (2014) New functionality of chalcogenide glasses for radiation sensing of nuclear wastes. J Hazard Mater 269:68-73. https://doi.org/10.1016/j.jhazmat.2013.11.050

14. Mahmud A, Gonzalez-Velo Y, Saremi M et al (2016) Flexible AgChG radiation sensors: limit of detection and dynamic range optimization through physical design tuning. IEEE Trans Nucl Sci 63:2137-2144. https://doi.org/10.1109/tns.2016.2555920

15. Kawaguchi T, Maruno S (1994) Photoinduced surface deposition of metallic silver: basic research for its application to optical recording devices. Jpn J Appl Phys 33:4521-4525. https://doi. org/10.1143/jjap.33.4521

16. Kawaguchi T, Maruno S (1994) Reversible Photowriting and thermal erasing of Ag patterns on Ag-rich Ag-Ge-S films. Jpn J Appl Phys 33:6470-6474. https://doi.org/10.1143/jjap.33.6470

17. Han JH, Jeong K-S, Ahn M, Lim DH, Yang WJ, Park SJ, Cho MH (2017) Modulation of phase change characteristics in Ag-incorporated $\mathrm{Ge}_{2} \mathrm{Sb}_{2} \mathrm{Te}_{5}$ owing to changes in structural distortion and bond strength. J Mater Chem C 5:3973-3982. https://doi. org/10.1039/c6tc05412a

18. Ji X, Song L, He W, Huang K, Yan Z, Zhong S, Zhang Y, Zhao R (2018) Super nonlinear electrodeposition-diffusion-controlled thin-film selector. ACS Appl Mater Interfaces 10:10165-10172. https://doi.org/10.1021/acsami.7b17235

19. Zhang S, Wu L, Song Z, Li T, Chen X, Yan S, Zhu M (2020) Breakthrough in high ON-state current based on $\mathrm{Ag}-\mathrm{GeTe}_{8}$ selectors. J Mater Chem C 8:2517-2524. https://doi.org/10.1039/c9tc06673j

20. Healy B, Merwe DVD, Christaki K, Meghzifene A (2017) Cobalt-60 machines and medical linear accelerators: competing technologies for external beam radiotherapy. Clin Oncol 29:110-115. https://doi.org/10.1016/j.clon.2016.11.002

21. Imanishi Y, Kida S, Nakaoka T (2016) Direct observation of Ag filament growth and unconventional SET-RESET operation in GeTe amorphous films. AIP Adv 6:075003. https://doi. org/10.1063/1.4958633

22. Imanishi Y, Hayashi H, Nakaoka T (2018) Spontaneous roomtemperature formation of broccoli-like $\mathrm{Ag}$-GeTe nanostructures assisting filamentary resistive switching. J Mater Sci 53:1225412264. https://doi.org/10.1007/s10853-018-2493-z

23. Bürgermeister $A$, Sitte $W$ (2001) Chemical diffusion in $\beta-\mathrm{Ag}_{2} \mathrm{Te}$. Solid State Ionics 141-142:331-334. https://doi.org/10.1016/ s0167-2738(01)00745-7

24. Wood C, Harrap V, Kane WM (1961) Degeneracy in $\mathrm{Ag}_{2}$ Te. Phys Rev 121:978-982. https://doi.org/10.1103/physrev.121.978

25. Prasai K, Chen G, Drabold DA (2017) Amorphous to amorphous insulator-metal transition in $\mathrm{GeSe}_{3}$ :Ag glasses. Phys Rev Mater 1:015603. https://doi.org/10.1103/physrevmaterials.1.015603

26. Xu L, Li Y, Yu NN, Zhong YP, Miao XS (2015) Local order origin of thermal stability enhancement in amorphous Ag doping GeTe. Appl Phys Lett 106:031904. https://doi.org/10.1063/1.4906332

27. Ailavajhala MS, Gonzalez-Velo Y, Poweleit C, Barnaby $\mathrm{H}$, Kozicki MN, Holbert K, Butt DP, Mitkova M (2014) Gamma radiation induced effects in floppy and rigid Ge-containing 
chalcogenide thin films. J Appl Phys 115:043502. https://doi. org/10.1063/1.4862561

28. Imanishi Y, Nakaoka T (2019) Direct measurement of 'readymade' cations in a Ge2Sb3.4Te6.2 film. J Mater Sci 54:7072-7077. https://doi.org/10.1007/s10853-019-03405-y

29. Khurana P, Vohra A, Srivastava KK (1990) Silver-doping effects on electrical properties of Ge-Te alloy. J Mater Sci Mater Electron 1:175-181. https://doi.org/10.1007/bf00696072

30. Serghei A, Tress M, Sangoro JR, Kremer F (2009) Electrode polarization and charge transport at solid interfaces. Phys Rev B. https ://doi.org/10.1103/physrevb.80.184301

31. Patil DS, Shimakawa K, Zima V, Wagner T (2014) Quantitative impedance analysis of solid ionic conductors: effects of electrode polarization. J Appl Phys 115:143707. https://doi. org/10.1063/1.4871286

32. Taibl S, Fafilek G, Fleig J (2016) Impedance spectra of Fe-doped SrTiO3thin films upon bias voltage: inductive loops as a trace of ion motion. Nanoscale 8:13954-13966. https://doi.org/10.1039/ c6nr00814c

33. Sulekar SS, Ordonez JE, Arango IC, Gomez MEP, Nino JC (2019) Effect of a DC bias on the conductivity of gadolinia doped ceria thin films. Electrochim Acta 303:275-283. https://doi. org/10.1016/j.electacta.2019.02.073

34. Saremi M (2016) A physical-based simulation for the dynamic behavior of photodoping mechanism in chalcogenide materials used in the lateral programmable metallization cells. Solid State lonics 290:1-5. https://doi.org/10.1016/j.ssi.2016.04.002

35. Perumal S, Roychowdhury S, Negi DS et al (2015) High thermoelectric performance and enhanced mechanical stability of p-type $\mathrm{Ge}_{1-\mathrm{x}} \mathrm{Sb}_{\mathrm{x}} \mathrm{Te}$. Chem Mater 27:7171-7178. https://doi. org/10.1021/acs.chemmater.5b03434

36. Noman MAA, Abden MJ, Islam MA (2018) Germanium telluride absorber layer, a proposal for low illumination photovoltaic application using AMPS 1D. 2018 International Conference on Computer, Communication, Chemical, Material and Electronic Engineering (IC4ME2), Rajshahi, pp 1-5

37. Goldmann A (2003) 2.9.3 Ag (Silver) $(Z=47)$. In SpringerMaterials -Landolt-Börnstein Database, ed. Goldmann A. http://mater ials.springer.com/lb/docs/sm_lbs_978-3-540-46039-8_4. https ://doi.org/10.1007/10714318_4
38. Karakaya I, Thompson W (1991) The Ag-Te (Silver-Tellurium) system. J Phase Equilib 12:56-63. https://doi.org/10.1007/bf026 63676

39. Gierlotka W (2009) Thermodynamic assessment of the AgTe binary system. J Alloys Compd 485:231-235. https://doi. org/10.1016/j.jallcom.2009.06.028

40. Mott NF (1976) The increase in the conductivity of chalcogenide glasses by the addition of certain impurities. Philos Mag 34:1101-1108. https://doi.org/10.1080/00318087608227732

41. Street RA, Mott NF (1975) States in the gap in glassy semiconductors. Phys Rev Lett 35:1293-1296. https://doi.org/10.1103/ physrevlett.35.1293

42. Kastner M, Adler D, Fritzsche H (1976) Valence-alternation model for localized gap states in lone-pair semiconductors. Phys Rev Lett 37:1504-1507. https://doi.org/10.1103/physrevlett.37.1504

43. Levine E, Washburn J, Thomas G (1967) Diffusion-induced defects in silicon I. J Appl Phys 38:81-87. https://doi. org/10.1063/1.1709015

44. Nardone M, Simon M, Karpov IV, Karpov VG (2012) Electrical conduction in chalcogenide glasses of phase change memory. J Appl Phys 112:071101. https://doi.org/10.1063/1.4738746

45. Buniatian V, Martirosyan N, Vorobiev A, Gevorgian S (2011) Dielectric model of point charge defects in insulating paraelectric perovskites. J Appl Phys 110:094110. https://doi. org/10.1063/1.3660376

46. Matsunaga T, Fons $\mathrm{P}$, Kolobov AV, Tominaga J, Yamada N (2011) The order-disorder transition in GeTe: views from different length-scales. Appl Phys Lett 99:231907. https://doi. org/10.1063/1.3665067

47. Gervacio-Arciniega JJ, Prokhorov E, Espinoza-Beltrán FJ, Trapaga $G$ (2012) Characterization of local piezoelectric behavior of ferroelectric GeTe and $\mathrm{Ge}_{2} \mathrm{Sb}_{2} \mathrm{Te}_{5}$ thin films. J Appl Phys 112:052018. https://doi.org/10.1063/1.4746087

48. Boschker JE, Wang R, Calarco R (2017) GeTe: a simple compound blessed with a plethora of properties. CrystEngComm 19:53245335. https://doi.org/10.1039/c7ce01040k

49. Polking MJ, Urban JJ, Milliron DJ et al (2011) Size-dependent polar ordering in colloidal GeTe Nanocrystals. Nano Lett 11:1147-1152. https://doi.org/10.1021/nl104075v 